\title{
Intravenous Azithromycin Plus Ceftriaxone Followed by Oral Azithromycin for the Treatment of Inpatients With Community-Acquired Pneumonia: An Open-Label, Non-Comparative Multicenter Trial
}

\begin{abstract}
Fernando G. Rubio ${ }^{1}$, Clóvis A. Cunha ${ }^{2}$, Fernando L.C. Lundgren ${ }^{3}$, Maria P.J.S. Lima ${ }^{4}$, Paulo J.Z. Teixeira ${ }^{5}$, Julio C.A. Oliveira ${ }^{6}$, Valdir Golin $^{7}$, Waldo L.L.D. Mattos ${ }^{8}$, Herbert K. Mählmann ${ }^{9}$, Edson D. Moreira ${ }^{10}$, Jose R. Jardim ${ }^{11}$, Rodney L.F. Silva ${ }^{12}$ and Patricia H.B. Silva ${ }^{13}$ ${ }^{1}$ Hospital de Base of FUNFARME, São José do Rio Preto, SP; ${ }^{2}$ Nossa Senhora das Graças Hospital, Curitiba, PR; ${ }^{3}$ Otávio de Freitas General Hospital, Recife, PE; ${ }^{4}$ Catholic University of Campinas, Campus II, Department of Infectious Diseases, Campinas, SP; ${ }^{5}$ Irmandade da Santa Casa de Misericórdia of Porto Alegre, Porto Alegre, RS; ${ }^{6}$ University Hospital of Federal University of Juiz de Fora, Juiz de Fora, MG; ${ }^{7}$ Irmandade da Santa Casa de Misericórdia of São Paulo, São Paulo, SP; ${ }^{8}$ Nossa Senhora da Conceição Hospital, Porto Alegre, RS; ${ }^{9}$ General Hospital of Itapecerica da Serra - OSS Seconci, Itapecerica da Serra, SP; ${ }^{10}$ General Hospital Roberto Santos, Salvador, BA; ${ }^{11}$ Federal University of São Paulo, São Paulo, SP; ${ }^{12}$ Clinical Hospital de Clinicas - Federal University of Paraná, Curitiba, PR; ${ }^{13}$ Sanatorinhos Hospital - OSS - Ação Comunitária, São Paulo, SP; Brazil
\end{abstract}

\begin{abstract}
Community-Acquired Pneumonia (CAP) is a major public health problem. In Brazil it has been estimated that $2,000,000$ people are affected by CAP every year. Of those, 780,000 are admitted to hospital, and 30,000 have death as the outcome. This is an open-label, non-comparative study with the purpose of evaluating efficacy, safety, and tolerability levels of IV azithromycin (IVA) and IV ceftriaxone (IVC), followed by oral azithromycin (OA) for the treatment of inpatients with mild to severe CAP. Eighty-six patients (mean age $56.6 \pm 19.8$ ) were administered IVA $(500 \mathrm{mg} / \mathrm{day})$ and IVC (1g/day) for 2 to 5 days, followed by AO $(500 \mathrm{mg} /$ day) to complete a total of 10 days. At the end of treatment (EOT) and after 30 days (End of Study - EOS) the medication was evaluated clinically, microbiologically and for tolerability levels. Out of the total 86-patient population, $62 \mathbf{( 7 2 . 1 \% )}$ completed the study. At the end of treatment, $95.2 \%\left(\mathrm{CI}_{95}: \mathbf{8 8 . 9} \%-100 \%\right)$ reported cure or clinical improvement; at the end of the study, that figure was $88.9 \%\left(\mathrm{CI}_{95}: 74.1 \%-91.7 \%\right)$. Out of the 86 patients enrolled in the study, 15 were microbiologically evaluable for bacteriological response. Of those, 6 reported pathogen eradication at the end of therapy $(40 \%)$, and 8 reported presumed eradication (53.3\%). At end of study evaluation, 9 patients showed pathogen eradication (50\%), and 7 showed presumed eradication (38.89\%). Therefore, negative cultures were obtained from $93.3 \%$ of the patients at EOT, and from $88.9 \%$ at the end of the study. One patient $(6.67 \%$ of patient population) reported presumed microbiological resistance. At study end, 2 patients $(\mathbf{1 1 . 1 1 \% )}$ ) still reported undetermined culture. Uncontrollable vomiting and worsening pneumonia condition were reported by $2.3 \%$ of patients.

Discussion and Conclusion Treatment based on the administration of IV azithromycin associated to ceftriaxone and followed by oral azithromycin proved to be efficacious and well-tolerated in the treatment of Brazilian inpatients with CAP.
\end{abstract}

Key-Words: Community acquired infections, pneumonia, anti-bacterial agents, macrolide ketolides, azithromycin.

Community-Acquired Pneumonia (CAP) is a major public health problem. The estimated yearly incidence in the United States is 4 million cases [1]. Of those, 600,000 are admitted to hospital, with death being the outcome for up to $40 \%$ [2] and with costs reaching 23 billion dollars [3,4]. In Brazil, CAP affects 2 million people annually, being the second most common cause of hospital admittance: 780,000, with 30,000 having death as the outcome. Appropriate microbiological exams identify infection-causing microorganisms in less than $50 \%$ of cases $[5,6]$. Therefore, the administration of antibiotics is based on results from clinical and epidemiological studies, being eminently empirical and usually administered to fight Streptococcus pneumoniae and Haemophilus influenzae: typically, the most frequently isolated microorganisms in adult patients admitted due to CAP. Pneumococcus is still the "number 1" pathogen in all age ranges, with penicillin

Received on 6 December 2007; revised 18 March 2008.

Address for correspondence: Dr. Fernando Rubio. Hospital de Base of FUNFARME. Av. Brigadeiro Faria Lima, 5416 - São José do Rio Preto - SP Brazil. Zip code: 15090-000. E-mail: Financial Support: gongora@famerp.brThe present study was conducted with the financial support of Pfizer.

The Brazilian Journal of Infectious Diseases 2008;12(3):202-209. (C) 2008 by The Brazilian Journal of Infectious Diseases and Contexto Publishing. All rights reserved. resistance variable at different geographical locations worldwide: in Brazil, it is approximately $2 \%-5 \%$, being typically presented as an invasive pneumococcic condition, which means that it is with bacteremia, empyema or concurrent meningitis.

Brandileone et al. carried out an evaluation through CLSI/ NCCLS clinical and laboratory interpretation endpoints for disc diffusion susceptibility and MIC results. Approximately $5.9 \%$ of pneumococci presented "high-level resistance", and approximately $22 \%$ presented "intermediate level resistance" [7].

CAP treatment is changeable, and tries to track down microorganisms. In 1993, the American Thoracic Society (ATS) published guidelines for the treatment of CAP. Those guidelines recommended that inpatients should receive empirical therapy based on the association of a beta-lactam and a macrolide [7], so that atypical agents (Mycoplasma, Chlamydophila pneumoniae (Chlamydia) and Legionella) could be covered. Atypical bacteria account for $15 \%-20 \%$ of hospital admittances due to CAP; therefore, in many cases, diagnosis is not predictable based on clinical and/or radiological conditions.

Studies have demonstrated that if that association is administered within 24 hours of admission, hospitalization 
time will be reduced by $50 \%$ [8,9]. Those recommendations were slightly changed in 2000, and confirmed in 2007, with the possibility of administering fluoroquinolones to inpatients with CAP as well as antibiotic therapy starting within 4 hours of admission at the latest [10].

In Brazil, the Brazilian Society of Pneumology and Tisiology published guidelines for the treatment of CAP in 2004. The guidelines recommend a systematic approach after the decision had been made as to whether treatment will be in hospital or through the outpatient unit. The guidelines set out that, inpatients on the ward must receive the association of a macrolide and a third generation cephalosporin or a macrolide associated to a betalactamic and to a beta-lactamase inhibitor (IV) or a fluoroquinolone [11-15]. However, the use of fluoroquinolones has been at the core of debates for the last 10 years due to its potential ability to induce resistance, as well as tolerability problems, especially among patients with comorbidities. That led the CDC to recommend use restriction for conditions requiring an empirical approach [16]. Although Brazil has never reported resistance of $S$. pneumoniae invasive strains to fluoroquinolones, fast-pace emergence of Gramnegative bacteria that were resistant to a number of antibiotic classes was observed at hospitals where fluoroquinolone administration was under no restriction in treating respiratory infections, which makes therapy management of infections acquired in that setting more difficult [17]. In February, 2007 Metersky et al. conducted a multivariate analysis of CAP treatment. They observed that the empirical use of fluoroquinolone may be harmful and does not impact on the mortality rate [18], as opposed to the association of a macrolide and a third generation cephalosporin, which impacts positively [19]. In Canada, S. pneumoniae resistant to quinolones was detected concurrent to increased use of fluoroquinolone to treat CAP [20]. The work mentioned earlier, by Brandileone and cols., showed that $6.2 \%$ of the 3,817 S. pneumoniae strains tested were erythromycin resistant. S. pneumoniae resistance to macrolides differs in the United States and in Europe. Major approaches to CAP treatment also differ. In North America macrolides are more widely used, especially due to the fact that pathogen resistance to macrolides is mediated through efflux pumps (low level), whereas in Europe the resistance process takes place through ribosomic site change (high level). The understanding of the macrolide efficacy and tolerability profile in different ethnic groups was turned into a key aspect in such complex scenarios.

A number of factors were taken into account for macrolide choice making. Azithromycin belongs to the azalide subclass in the macrolide antibiotic class, with a proven in vitro and in vivo action spectrum against major pneumonia-causing agents: $S$. pneumoniae, $H$. influenzae, C. pneumoniae, $L$. pneumophila, $M$. catarrhalis, $M$. pneumoniae and $S$. aureus (oxacillin sensitive). Since approval in the United States in 1997, azithromycin IV formulation has been widely used for inpatients with CAP. In addition to action spectrum, azithromycin pharmacokinetics and high tissue levels allow a single daily dose regimen. When CAP patients are treated in the outpatient unit, those pharmacological properties allow the use of azithromycin as monotherapy [21]. The purpose of the present study was to evaluate the efficacy, safety and tolerability of a combined regimen - azithromycin and ceftriaxone - both IV, followed by oral azithromycin when treating inpatients with moderate to severe CAP.

This is the first scientific paper in Brazil to show the efficacy of ceftriaxone + azithromycin to treat CAP in the Brazilian population scenario.

\section{Materials and Methods \\ Eligibility Criteria}

Both male and female patients over 18 years old, with history, clinical and radiological findings consistent with CPA diagnosis and whose severity required hospitalization and initial therapy with IV antibiotics were eligible to enroll in the study. The following criteria had to be met: (1) presence of alveolar infiltrate at thoracic X-ray, and at least two of the following findings within 48 hours prior to first visit (Visit I): onset of cough or worsening of chronic cough; change in sputum characteristics; axillary temperature $>38^{\circ} \mathrm{C}$ (fever) or $<36.1^{\circ} \mathrm{C}$ (hypothermia) in the previous 24 hours; pulmonary auscultation compatible with consolidation (fine bubbling rale); and leukocytosis (leukocytes $>10,000 / \mathrm{mm}^{3}$ or $>15 \%$ young leukocytes); (2) presence of any score in severity criteria for coexistent diseases in the Pneumonia Severity Index (PSI) or any PSI score that would classify a patient as $\geq$ II [22]. Pregnant or lactating women patients were excluded, as well as women of childbearing age not using effective contraceptives. Also excluded were patients presenting the following conditions/treatment: allergies or known or suspected intolerance to penicillin, cephalosporin, or macrolides; systemic treatment with any antibiotic for any period longer than 24 hours prior to first visit, or longer than seven days in the previous month; AIDS patients, neutropenics, (leukocytes $<2,500 / \mathrm{mm}^{3}$ or neutrophils $<1,000 /$ $\mathrm{mm}^{3}$ ) or undergoing chronic immunosuppressant therapy; clinical or radiological signs of pulmonary cavitation; cystic fibrosis, primary or metastatic pulmonary neoplasia, aspiration pneumonia, pleural empyema, tuberculosis or bronchiecstasis; gastrointestinal disorders or any other condition affecting the drug under investigation; epilepsy or seizures; clinically significant renal failure - creatinine $>2.0 \mathrm{mg} / \mathrm{dL}$, or estimated creatinine clearance $<20 \mathrm{~mL} / \mathrm{min} / 1.73 \mathrm{~m}^{2}$ of body surface area; evidence of clinically significant hepatic failure with transaminase levels three times above the normal upper limit, or total bilirubin levels twice the upper normal limit; clinically significant cardiovascular condition. Patients participating in any other study involving investigational or commercial drugs concurrently or having participated within a month before the start of the present study were also excluded, as were those who had previously participated in the present study, those with evidence of recent alcohol or illegal drug abuse; and those who presented any condition which, following

www.bjid.com.br 
investigators' discretion, could interfere in their safety, their treatment response assessment, or that could in any way preclude treatment completion or follow-up; and those who had donated blood less than four weeks before study starting date or who planned to donate blood within a time period of four weeks after study completion. Childbearing age women who had not been surgically sterilized had to undergo a pregnancy test immediately before the study started. Additionally, effective contraceptive methods were expected to be in use during treatment period and for 10 days after study close. All patients included in the study signed the informed consent form. Study protocol was pre-approved by the Research Ethics Committee at each of the 13 institutions participating in the study. The study was conducted in compliance with Good Clinical Research Practices, the ethic principles of the Declaration of Helsinki, and the Brazilian legislation with regard to clinical research.

\section{Study Design}

This was a 30-day open-label, multicenter, non-comparative study. The study drug was administered for a period of 10 days. The average number of patients per center was 7 . The center with the highest number of patients had a total of 30 enrollments. CAP patients who fulfilled the eligibility criteria were enrolled in the study. All patients received a single daily dose of $500 \mathrm{mg}$ of azithromycin associated to $1 \mathrm{~g}$ of ceftriaxone (IV) for a period of between 2 and 5 days. Treatment proceeded with a single daily dose of $500 \mathrm{mg}$ of oral azithromycin to complete 10 treatment days. For cases where S. pneumoniae resistant to a macrolide was isolated in culture, the association of cefuroxime axetil and oral azithromycin was allowed. Patients were evaluated at enrollment (Visit I), on Visit II (day 3), at the end of treatment (Visit III - day 10-12), and by study completion (Visit IV - day 28 to 35), as well as daily during hospitalization. Clinical and bacteriological safety assessment was carried out at Visits I, III, and IV. Patients who finished treatment were still evaluated for safety for a period of 30 days. On Visit I the following data were collected: demographics, comorbidity history, information on the use of antibiotics in the previous month, medications currently being used, and information from an objective physical exam, which included measures of vital signs (heart and respiratory rate, blood pressure and temperature). Clinical evaluation of pneumonia signs and symptoms involved sputum characteristics, cough, dyspnea, pleuritic pain, rigors and adventitious sounds at pulmonary auscultation. Two chest X-Rays were taken: frontal and lateral view. PSI determination criteria were also evaluated, which resulted in a PSI score [22]. Blood and urine samples were collected for safety laboratory analysis. Childbearing age female patients were submitted to pregnancy test (urine or blood). Whenever possible, proper sputum samples - defined as a sample with $>25$ polymorphonuclear leukocytes and $<$ than 10 epithelial cells per $100 \mathrm{x}$ field after Gram's stain test - and blood samples were obtained for culture. The sputum samples were submitted to microbiological qualitative evaluation only. Those showing bacterial growth were submitted to an antibiogram and tested locally at the center's laboratory with regard to antibiotic susceptibility following the National Committee for Clinical Laboratory Standards criteria. All patients enrolled in the study had blood samples taken for hemoculture locally at the center's laboratory. During the same visit urine samples were collected to detect Streptococcus pneumoniae and Legionella. Ideally, three sputum samples and hemoculture should have been collected for evaluation.

On Visit II (day3) the need for IV antibiotic therapy was assessed. The change to oral therapy was seen as appropriate whenever all of the criteria below were present: (1) body temperature $<37.8^{\circ} \mathrm{C}$ for a minimum of 8 hours; (2) cough and dyspnea condition improvement; (3) proper oral intake and absorption; (4) leukocytosis reduction as compared to baseline. All signs and symptoms identified in Visit I were reevaluated. New ones were reported. Hematological and biochemical laboratory exams were repeated. Whenever possible, a second proper sample was obtained for sputum culture. If no expectoration was present even after nebulization and physiotherapy, culture was considered negative. Hemoculture collection was repeated if Visit I collection was positive, or if the patient had been discontinued from the study due to therapeutic failure.

At the end of treatment (Visit III, day 10 to 12), global clinical response to treatment was evaluated and classified as cure, improvement or therapeutic failure. Each of them were defined as follows: (1) cure: the resolution of signs and symptoms, with clinical recovery to before the occurrence of pneumonia condition, which included leukocytosis reduction, with improvement or non-progression of infiltrates at thoracic X-Ray; (2) improvement, as resolution of fever, but incomplete resolution of other signs and symptoms, with no need for additional antibiotic therapy; (3) therapeutic failure, such as no resolution or worsening of any of pneumonia signs or symptoms, with the need for additional antibiotic therapy. Therapeutic failure might be associated to the antibiotic - in the case that the patient had been administered the study drug for at least 48 hours - or non-related to the antibiotic - in the case that the patient had been administered the drug for less than 48 hours; (4) undetermined - when patient response to treatment could not be evaluated (lost due to follow-up or study withdrawal, except when due to therapeutic failure, among other reasons). At study close (Visit IV, days 28 to 35) or at early discontinuation, the investigator conducted a final evaluation of the global clinical response to treatment. Classification was: cure, improvement, or therapeutic failure. Both, signs and symptoms that were identified at earlier visits, and new ones that might have emerged, were compared. Those signs and symptoms were all compared to Visit I. Vital signs were also evaluated, as well as concurrent use of other medications and the occurrence of adverse events. Hematological and biochemical exams were repeated only if a clinically significant adverse event occurred, or if any relevant 
laboratory change was detected at Visit III. A final thoracic Xray was taken, and whenever possible, a proper sample was obtained for sputum culture. If no sample could be obtained, it was considered equivalent to a negative culture. If initial hemoculture was positive, or if the patient was discontinued due to clinical failure, a new sample was collected for blood culture.

\section{Efficacy and Safety Evaluation}

The sample from intention to treat (ITT) patients included those who received at least one dose of IV azithromycin, and who provided sufficient data for at least one efficacy parameter analysis, conducted separately from clinical and bacteriological response. Primary efficacy parameter was clinical response, defined as the percentage of cure or improvement at the end of the study. Secondary efficacy parameters were clinical response at the end of the study and bacteriological response rate at the end of treatment (Visit III) and at end of the study (Visit IV). Clinical response was determined by comparing clinical presentation global evaluation at study end and Visit I. Bacteriological response was evaluated at the end of treatment (Visit III) and at the end of study (Visit IV), having been defined as follows: (1) eradication, when the isolated pathogen was eliminated early, in same site sample (sputum or blood); (2) presumed eradication, in the absence of a proper sputum sample, associated to clinical improvement; (3) persistence, when the eradication of the infection causing agent was not achieved; (4) relapse, when the pathogen isolated at Visit I was again present within 5 days of treatment discontinuation, or during treatment, after a second negative culture; (5) super infection, a new infection in the lower respiratory tract - documented by thoracic X-Ray or pulmonary auscultation, during treatment or up to three days after close of treatment - caused by a new pathogen or by a resistant pathogen; (6) colonization, when sputum culture was positive for a bacteria that was different from that isolated in the first 48 hours of treatment as the causal agent, and persisting for at least two other cultures, not associated with a new infection or clinical condition; (7) eradication with infection, when the initial pathogen was eliminated, followed by the isolation of a new pathogen or another serotype of the same microorganism, with infection signs at the end of treatment; (8) presumed persistence, defined as concurrent use of antibiotic therapy due to persistent clinical symptoms and signs of infection, in the absence of microbiological data; (9) undetermined, defined as the absence of pathogen isolation in the initial sample, absence of culture collection after Visit I, or patient not clinically evaluable. Safety analysis was conducted in the sample of patients that received at least one dose of the study drug (safety population). All adverse events were noted throughout the study. Serious adverse events were defined as any occurrence that might have put the patient's life at risk or resulted in hospitalization, hospitalization extension, persistent or significant impairment, teratogeny, or death. Safety analysis parameters were evaluated throughout the study and were based on the adverse event rate, early discontinuation, laboratory changes, physical exam, and vital signs.

\section{Statistical Analysis}

Sample size was estimated based on cure rate at the end of the study: $75 \%$. Two-sided $95 \%$ CI for that rate with $20 \%$ width could be obtained with a 73-patient sample. Assuming loss to follow-up or non-evaluation of up to $20 \%$ of patients, an 88-patient sample size would be required. Patient ratio for each clinical and bacteriological response category was obtained. A 95\% CI, based on the normal approximation to the binomial distribution, was calculated.

\section{Results}

\section{Study Population}

A total of 88 patients were selected, of those, 86 received the proposed treatment. Forty five patients were male (51.2\%) and approximately $66 \%$ were white. Age range was $56.6 \pm 19.8$, and average time from onset of pneumonia was 6.7 days ( 1 to 22 days). PSI mean score was $82.0 \pm 26.2$, ranging from 28.0 to 143.0. Most commonly found comorbidities were congestive heart failure (15 patients), diabetes mellitus (14 patients), chronic pulmonary obstructive disease (COPD) (13 patients) and asthma (6 patients). Data on patients enrolled in the study are summarized in Table 1.

Table 1. Patients' data at study starting point $(\mathrm{N}=86)$

\begin{tabular}{lc}
\hline Variable & $\mathbf{N ~ ( \% )}$ \\
\hline Gender & \\
$\quad$ Female & $42(48.8)$ \\
$\quad$ Male & $44(51.2)$ \\
Age (years) & \\
$\quad$ Mean \pm SD & $56.6 \pm 19.8$ \\
$\quad$ Range & $17-93$ \\
Race & \\
$\quad$ White & $56(65.1)$ \\
$\quad$ Black & $14(16.3)$ \\
$\quad$ Mixed & $16(18.6)$ \\
Comorbidities & \\
$\quad$ Congestive heart failure & $15(17.4)$ \\
Diabetes mellitus & $14(16.3)$ \\
$\quad$ Chronic pulmonary obstructive & \\
$\quad$ disease (COPD) & $13(15.1)$ \\
$\quad$ Asthma & $6(7.0)$ \\
Time from onset of symptoms (days) & \\
$\quad$ Mean ( range) & $6.7(1-22)$ \\
Pneumonia severity index (PSI) & $82.0 \pm 26.2$ \\
$\quad$ Mean \pm SD & $28-143$ \\
Range &
\end{tabular}

Of the 86 patients who were treated with the study drugs $62(72.1 \%)$ reached study completion. A total of 24 patients (27.9\%) did not reach completion for the following reasons: adverse events (13 patients - 15.1\%); change in laboratory 
testing (1 patient - 1.2\%); no efficacy (1 patient $-1.2 \%)$; lost to follow-up (1 patient $-1.2 \%)$; sudden death (1 patient $1.2 \%)$; other reasons ( 7 patients $-8.1 \%$ ). A total of 85 patients were included in the Modified Intention to Treat (MITT) population, made up of all patients that had been treated, except those who were wrongly diagnosed or did not present clinical symptoms at evaluation 1. 24 patients reported microbiological data and were analyzed with reference to bacteriological efficacy parameters.

\section{Treatment Efficacy Evaluation}

When the study started, pneumonia signs and symptoms were presented as follows: cough - 95.3\%; dyspnea - 84.7\%; pulmonary auscultation with rale $-89.4 \%$; pleuritic pain $64.7 \%$; snoring at pulmonary auscultation $-28.2 \%$; wheezing $-27.1 \%$ and rigors $-27.1 \%$. Pneumonia signs and symptoms showed significant improvement at the end of treatment: cough $-37.1 \%$; rale $-16.1 \%$; pleuritic pain $-9.7 \%$; snoring $-6.5 \%$ and dyspnea $-6.5 \%$.

The number of patients who reported a positive clinical response (cure or improvement) can be found in Table 2. The number of patients who reported a positive bacteriological response (eradication plus presumed eradication) is shown in Table 3. Twenty four patients (27.9\% of the study population) made up the population that was modified for bacteriological evaluation through hemoculture or sputum. The latter reported a $6.67 \%$ and $11.11 \%$ rate of pneumococci that were resistant to macrolides at the end of treatment and at the end of study respectively. Only 1 patient used cefuroxime axetil rather than azithromycin.

Fifteen patients were evaluated at the end of treatment. Of those, $40 \%$ reported eradication, $53.3 \%$ reported presumed eradication, and $6.7 \%$ reported presumed persistence.

At close of study out of the 18 patients that were evaluable through microorganism isolation (sputum or hemoculture) $88.9 \%$ reported eradication or presumed eradication. (Table 3 )

Table 4 shows clinical response to base pathogen in the MITT population in patients whose pathogen was isolated. Evaluation of pneumonia etiological agents that were identified was as follows: at the end of the therapy, from patients infected by Streptococcus pneumoniae $77.78 \%$ reported cure ( 7 patients) and 22.22\% reported improvement (2 patients); of those infected by Haemophilus influenza $100 \%$ reported improvement (2 patients); of those infected by cure (9 patients) reported cure and $14.29 \%$ reported failure. Of the 62 patients, 18 had etiological agents identified, as listed below.

\section{Safety and Tolerability Evaluation}

Of the 86 patients in the safety population, 64 patients (74.4\%) reported adverse events. Of those, the adverse events reported by 33 patients (38.4\%) were related to the study drug. A total of 141 adverse events were observed. Of those, 55 (39\%) were related to the study drug. Serious adverse events were reported by 20 patients, but only two patients had their serious adverse events - pneumonia condition worsening and uncontrollable vomiting - related to azithromycin/ ceftriaxone. Both patients were discontinued from the study. Of the 14 patients (16.3\%) that were discontinued from the study due to adverse events, 5 (5.8\%) were discontinued due to treatment-related adverse events. Most commonly found adverse events were: no therapeutic response in 10 patients (11.6\%); pneumonia persistence in 7 patients (8.1\%); increase in transaminase levels in 6 patients (7.0\%); pain at infusion site in 5 patients (5.8\%); epigastralgia in 5 patients (5.8\%); diarrhea in 5 patients (5.8\%); vomiting in 5 patients (5.8\%) and hypertension in 5 patients (5.8\%). A more detailed description of drug-related adverse events and their severity can be found in Table 5 .

\section{Discussion}

Community Acquired Pneumonia is a high prevalence condition, with a significant morbimortality rate and approximately $20 \%$ of cases resulting in hospital admittance [23].

The present study confirms that IV administration of azithromycin associated to ceftriaxone and followed by oral azithromycin has shown good clinical efficacy in treating CAP inpatients. The possibility of changing to oral administration after between 48 and 72 hours of parenteral treatment may result in shorter hospital stay time.

Current studies support the macrolide - betalactamic association. A number of studies of the treatment of CAP recommend coverage for atypical agents as well as $S$. pneumoniae, including Mycoplasma, Chlamydophila pneumoniae (Chlamydia) and Legionella. Many Mycoplasma or Chlamydophila pneumoniae (Chlamydia) infections are self-limiting or are considered mixed infections that may respond to betalactamics. Subgroup analysis in Legionella infected patients shows that the therapeutic failure rate is significantly lower in patients treated with antibiotics with coverage for atypical agents.

Shefet et al. have conducted an analysis of 32 recent studies involving 918 CAP patients who were treated with amoxicillin, 2,940 treated with fluoroquinolone, and 2,018 treated with clarithromycin and azithromycin. Corresponding rates demonstrated for clinical response were: $80 \%, 88 \%$, $87.5 \%$. Clinical response rates for treatment using fluoroquinolones and macrolides were significantly higher when compared to those using amoxicillin.

That makes the present study's findings more material. The data also show that for every 12 CAP patients treated with amoxicillin 1 therapeutic failure was reported - and it could have been prevented if a macrolide or a fluoroquinolone [27] had been used.

A number of studies have tried to measure the impact of treatment compliance for CAP outcome. However, none of those studies were designed to evaluate specific antibiotic therapy regimens.

The present study is not failure free. Major criticism may be raised of the qualitative - rather than quantitative microbiological analysis, as well as of the fact that cultures 
Table 2. Number of patients who presented a positive response to treatment with IV azithromycin followed by oral azithromycin at day 10 of treatment (Visit III) and at the end of study (Visit IV).

\begin{tabular}{lcc}
\hline Clinical Response & Visit III & Visit IV \\
\hline Patients evaluated & $62(100.0 \%)$ & $70(100.0 \%)$ \\
Cure & $42(67.7 \%)$ & $58(82.8 \%)$ \\
Improvement & $17(27.4 \%)$ & 0 \\
Failure & $3(4.8 \%)$ & $12(17.1 \%)$ \\
Positive clinical response (cure or improvement) & $59(95.2 \%)$ & $58(82.9 \%)$ \\
& $\mathrm{CI}_{95 \%}[88.9-100]$ & $\mathrm{CI}_{95 \%}[74.1-91.7]$ \\
\hline
\end{tabular}

Table 3. Bacteriological response distribution in patients reporting isolated microorganisms in sputum culture or hemoculture at study start.

\begin{tabular}{llr}
\hline & Bacteriological response & N (\%) \\
\hline Treatment end (Visit III) & Patients evaluated & $15(100.0 \%)$ \\
& Eradication & $6(40.0 \%)$ \\
& Presumed Eradication & $8(53.3 \%)$ \\
Study end (Visit IV) & Presumed Persistence & $16.7 \%)$ \\
& Patients evaluated & $18(100.0 \%)$ \\
& Eradication & $9(50.0 \%)$ \\
& Presumed Eradication & $7(38.9 \%)$ \\
& Undetermined & $2(11.1 \%)$ \\
\hline
\end{tabular}

Table 4. Clinical response to base pathogen - bacteriological MITT population.

\begin{tabular}{llll}
\hline Pathogen & Visit & Clinical response & Azithromycin \\
\hline $\begin{array}{l}\text { Streptococcus pneumoniae } \\
\text { Streptococcus pneumoniae }\end{array}$ & End of therapy (Visit III) & Cure improvement & $7(77.78 \%) 2(22.22 \%)$ \\
& End of study (Visit IV) & Cure/failure/undetermined & $9(75 \%) 1(8.33 \%)$ \\
Haemophilus influenzae & End of therapy (Visit III) & Improvement & $2(16.67 \%)$ \\
Haemophilus influenzae & End of study (Visit IV) & Cure & $2(100 \%)$ \\
Staphylococcus aureus & End of therapy (Visit III) & Cure & $2(100 \%)$ \\
Staphylococcus aureus & End of study (Visit IV) & Cure & $1(100 \%)$ \\
Other pathogens & End of therapy (Visit III) & Cure/improvement/failure & $1(100 \%)$ \\
& & & $4(57.14 \%) 2(28.57 \%)$ \\
Other pathogens & End of study (Visit IV) & Cure/failure & $1(14.29 \%)$ \\
\hline
\end{tabular}

were not presented by collection site (hemoculture or sputum), but by patient (eradication or persistence).

Some conclusions may be drawn, however, to be added to the literature currently available.

Retrospective conventional studies suggest that treatment using a macrolide combined, typically with a cephalosporin, to form an initial therapeutic scheme for patients who need hospitalization may be associated to lower mortality rate and shorter hospital stay time when compared to treatment with cephalosporin only [30,31].

Sanchez et al. conducted a prospective study in 2003 involving elderly patients with CAP. In the study, IV administration of azithromycin and clarithromycin associated to ceftriaxone was compared. Hospital stay time was $7.4 \pm$ 5.0 days for the group treated with azithromycin, and $9.4 \pm$
7.0 days for the group treated with clarithromycin $(\mathrm{p}<0.01)$. The mortality rate was also lower in the azithromycin group when compared to the clarithromycin group (3.6\% vs. 7.2\%; $\mathrm{p}<0.05)$. These data suggest the association azithromycinceftriaxone to be more efficacious when compared to clarithromycin [34].

The present study has shown that the combination of IV azithromycin and ceftriaxone to treat CAP inpatients agreed with previous publications, having shown $95.2 \%$ clinical response at the end of treatment, and $82.9 \%$ at the end of the study $[31,35,36]$. Approximately $75 \%$ of patients reported some adverse event during the study, but only $38.4 \%$ were related to azithromycin. From all serious adverse events only 2 were related to the study drug, and resulted in the withdrawal of the two patients from the study. This data is in 
Table 5. Incidence and severity of treatment-related adverse events.

\begin{tabular}{lcccc}
\hline \multicolumn{4}{c}{$\begin{array}{c}\text { Number of subjects evaluable for adverse events } \\
\text { azithromycin/ceftriaxone } \\
(\mathbf{n}=\mathbf{8 6})\end{array}$} & \\
\hline Event & $\mathbf{N}$ & Mild & Moderate & Severe \\
\hline Blood and lymphatic system disorders & $1(1.2)$ & 1 & 0 & 0 \\
$\quad$ Leucopenia & $1(1.2)$ & 1 & 0 & 0 \\
Genetic, congenital and family disorders & $1(1.2)$ & 1 & 0 & 0 \\
Fissured tongue, congenital & $1(1.2)$ & 1 & 0 & 0 \\
Gastrointestinal disorders & $13(15.1)$ & 10 & 3 & 0 \\
Upper abdominal pain & $4(4.7)$ & 3 & 1 & 0 \\
Aphthous stomatitis & $1(1.2)$ & 1 & 0 & 0 \\
Constipation & $1(1.2)$ & 1 & 0 & 0 \\
Diarrhea & $4(4.7)$ & 3 & 1 & 0 \\
Dyspepsy & $1(1.2)$ & 1 & 0 & 0 \\
Nausea & $1(1.2)$ & 0 & 1 & 0 \\
Vomiting & $4(4.7)$ & 3 & 1 & 0 \\
General disorders and infusion site conditions & $11(12.8)$ & 7 & 3 & 1 \\
Burning at infusion site & $2(2.3)$ & 2 & 0 & 0 \\
Pain at infusion site & $4(4.7)$ & 3 & 0 & 1 \\
Therapeutic response failure & $6(7.0)$ & 3 & 3 & 0 \\
\hline
\end{tabular}

agreement with current literature, and has demonstrated the concern with regard to pharmacosurveillance in the present study.

In conclusion: azithromycin presents a good safety and tolerability profile. Therefore, IV azithromycin associated to ceftriaxone may be an appropriate therapeutic choice to treat CAP patients who need hospitalization in Brazil. Currently, the most widely accepted concept with regard to the starting dose time frame for CAP patients who need hospitalization is "the more septic the patient, the more likely it is that as little as a 1 hour delay may impact mortality rate”. The recommendation is that the first dose be administered at the Emergency Service where the patient is seen for the first time, rather than waiting for the patient to be admitted. That would mean good conduct assistance and the best option choice for the patient.

\section{References}

1. Bartlett J.G., Mundy L.M. Community-acquired pneumonia. N Engl J Med 1995;333(24):1618-24.

2. Fine M.J., Smith M.A., Carson C.A., et al. Prognosis and outcomes of patients with community-acquired pneumonia. A metaanalysis. JAMA 1996;275(2):134-41.

3. Marrie T.J., Peeling R.W., Fine M.J., et al. Ambulatory patients with community-acquired pneumonia: the frequency of atypical agents and clinical course. Am J Med 1996;101(5):508-15.

4. Marrie T.J. Community-acquired pneumonia. Clin Infect Dis 1994;18(4):501-15.

5. Bates J.H., Campbell G.D., Barron A.L., et al. Microbial etiology of acute pneumonia in hospitalized patients. Chest 1992;101(4):1005-12.

6. Fang G.D., Fine M., Orloff J., et al. New and emerging etiologies for community-acquired pneumonia with implications for therapy. A prospective multicenter study of 359 cases. Medicine (Baltimore) 1990;69(5):307-16.
7. Brandileone M.C., et al. Increase in numbers of beta-lactamresistant invasive Streptococcus pneumoniae in Brazil and the impact of conjugate vaccine coverage. Journal of Medical Microbiology 2006;55:567-74.

8. ATS Statement. Guidelines for the initial management of adults with conmunity-acquired pneumonia: diagnosis assesment of severity, and initial antimicrobial therapy. Am Rev Respir Dis 1993; $148: 1418-26$.

9. Stahl J.E., Barza M., Desjardin J., et al. Effect of macrolides as part of initial empiric therapy on length of stay in patients hospitalized with community-acquired pneumonia. Arch Intern Med 1999;159:2576-80.

10. Lode H. In: Azithromycin in the treatment of community-acquired pneumonia in adults. Cambridge Medical Publications, 2002.

11. Sanchez F., Mensa J., Martinez J.A., et al. Is Azithromycin the First-Choice Macrolide for Treatment of Community-Acquired Pneumonia? Clin Infect Dis 2003;6(10):1239-45.

12. Plouffe J., Schwartz D.B., Kolokathis A., et al. Clinical efficacy of intravenous followed by oral azithromycin monotherapy in hospitalized patients with communityacquired pneumonia. The Azithromycin Intravenous Clinical Trials Group. Antimicrob Agents Chemother 2000;44(7):1796-802.

13. American Thoracic Society. Guidelines for the initial management of adults with community-acquired pneumonia: diagnosis, assesment of severity, and initial antimicrobial therapy. Am Respir Crit Care Med 2001;163:1730-54.

14. Pereira C.A.C., Rocha R.T., Silva R.F., et al. Diretrizes para Pneumonias Adquiridas na Comunidade (PAC) em Adultos Imunocompetentes. J Bras Pnem 2004;30(Supl)4:11-4.

15. Bartlett J.G., Dowell S.F., Mandell L.A., et al. Practice guidelines for the management of community-acquired pneumonia in adults. Infectious Diseases Society of America. Clin Infect Dis 2000;31(2):347-82.

16. Mandell L.A., Wunderink R.G., Anzueto A., et al. Infectious Diseases Society of America/American Thoracic Society Consensus Guidelines on the Management of CommunityAcquired Pneumonia in Adults. Clin Infect Dis 2007;44 Suppl 2:S27-72. 
17. Heffelfinger J.D., Dowell S.F., Jorgensen J.H., et al. Management of community-acquired pneumonia in the era of pneumococcal resistance: a report from the Drug-Resistant Streptococcus pneumoniae Therapeutic Working Group. Arch Intern Med 2000;160(10):1399-8.

18. Rifenburg R.P., Paladino J.A., Bhavnani S.M., et al. Influence of fluoroquinolone purchasing patterns on antimicrobial expenditures and Pseudomonas aeruginosa susceptibility. Am J Health Syst Pharm 1999;56(21):2217-23.

19. Park-Wyllie L.Y., Juurlink D.N., Kopp A., et al. Outpatient gatifloxacin therapy and dysglycemia in older adults. N Engl J Med 2006;354:1352-61.

20. Metersky M.L., Ma A., Houck P.M., Bratzler D.W. Antibiotics for bacteremic pneumonia. Chest 2007;131:466-73.

21. Chen D.K., McGeer A., de Azavedo J.C., Low D.E. Decreased susceptibility of Streptococcus pneumoniae to fluoroquinolones in Canada. Canadian Bacterial Surveillance Network. N Engl J Med 1999;341(4):233-9.

22. Garey K.W., Amsden G.W. Intravenous azithromycin. Ann Pharmacother 1999;33(2):218.

23. Fine M.J., Singer D.E., Hanusa B.H., et al. Validation of a pneumonia prognostic index using the MedisGroups Comparative Hospital Database. Am J Med 1993;94(2):153-9.

24. Mandell L.A., Bartlett J.G., Dowell S.F., et al. Update of practice guidelines for the management of community-acquired pneumonia in immunocompetent adults. Clin Infect Dis 2003;37:1405.

25. File T.M. Community-acquired pneumonia. Lancet 2003;362:1991.

26. Mills G.D., Oehley M.R., Arrol B. Effectiveness of beta lactam antibiotics compared with antibiotics active against atypical pathogens in non-severe community acquired pneumonia: metaanalysis. BMJ 2005;330:456.

27. Shefet D., Robenshtock E., Paul M., Leibovici L. Empiric antibiotic coverage of atypical pathogens for community acquired pneumonia in hospitalized adults. Cochrane Database Syst Rev 2005:CD004418.
28. Mandell L.A., Marrie T.J., Grossman R.F., et al. Canadian guidelines for the initial management of community-acquired pneumonia: an evidence-based update by the Canadian infectious diseases society and the Canadian thoracic society. Clin Infect Dis 2000;31:383-421.

29. Mortensen E.M., Restrepo M., Anzueto A., Pugh J. Effects of guideline-concordant antimicrobial therapy on mortality among patients with community-acquired pneumonia. Am J Med 2004; $117: 726$

30. Menendez R., Torres A., Zalacain R., et al. Guidelines for the treatment of community-acquired pneumonia: Predictors of adherence and outcome. Am J Respir Crit Care Med 2005;172:757.

31. Stahl J.E., Barza M., DesJardin J., et al. Effect of macrolides as part of initial empiric therapy on length of stay in patients hospitalized with community-acquired pneumonia. Arch Intern Med 1999;159(21):2576-80

32. Gleason P.P., Meehan T.P., Fine J.M., et al. Associations between initial antimicrobial therapy and medical outcomes for hospitalized elderly patients with pneumonia. Arch Intern Med 1999;159(21):2562-72.

33. Martinez J.A., Horcajada J.P., Almela M., et al. Addition of a macrolide to a beta-lactam-based empiric antibiotic regimen is associated with lower in-hospital mortality for patients with bacteremic pneumococcal pneumonia. Clin Infect Dis 2003;36:389.

34. Waterer G.W., Somes G.W., Wunderink R.G. Monotherapy may be suboptimal for severe bacteremic pneumococcal pneumonia. Arch Intern Med 2001;161:1837.

35. Sanchez F., Mensa J., Martinez J.A., et al. Is azithromycin the first-choice macrolide for treatment of community-acquired pneumonia? Clin Infect Dis 2003;36(10):1239-45.

36. Bohte R., van't Wout J.W., Lobatto S., et al. Efficacy and safety of azithromycin versus benzylpenicillin or erythromycin in community-acquired pneumonia. Eur J Clin Microbiol Infect Dis 1995;14(3): 182-7.

37. Paladino J.A., Gudgel L.D., Forrest A., Niederman M.S. Costeffectiveness of IV-to-oral switch therapy: azithromycin vs. cefuroxime with or without erythromycin for the treatment of community-acquired pneumonia. Chest 2002;122(4):1271-9. 